\title{
Action publique et institution d'une branche professionnelle. Le cas de la course urbaine
}

Public Action and the Institution of a sector-level Bargaining. The case of courier Services

Marnix Dressen et Arnaud Mias

\section{OpenEdition}

\section{Journals}

Édition électronique

URL : http://journals.openedition.org/travailemploi/1968

DOI : 10.4000/travailemploi.1968

ISSN : 1775-416X

Éditeur

DARES - Ministère du Travail

Édition imprimée

Date de publication : 15 juin 2008

Pagination : 7-19

ISSN : 0224-4365

Référence électronique

Marnix Dressen et Arnaud Mias, « Action publique et institution d'une branche professionnelle. Le cas de la course urbaine », Travail et Emploi [En ligne], 114 | avril-juin 2008, mis en ligne le 05 novembre 2010, consulté le 09 avril 2021. URL : http://journals.openedition.org/travailemploi/1968; DOI : https://doi.org/10.4000/travailemploi.1968 


\title{
Action publique et institution d'une branche professionnelle. Le cas de la course urbaine
}

\author{
Marnix Dressen (*) Arnaud Mias(**)
}

\begin{abstract}
L'article s'attache à une analyse sociologique des relations professionnelles dans le secteur spécifique de la course urbaine en termes d'acteurs, à travers la description de la mise en place d'une régulation de branche à ce niveau. Dans un secteur caractérisé par un grand nombre de très petites entreprises peu régulées, où les organisations représentatives patronales ou salariées étaient, il y a une dizaine d'années, encore insuffisamment présentes, les auteurs décrivent la part spécifique prise par l'État, plus précisément, par certains fonctionnaires de l'administration des transports. Audelà des approches habituelles qui s'inspirent de la théorie de la régulation sociale de J.-D. Reynaud, les auteurs font appel, pour comprendre les dynamiques entre État et acteurs sociaux, et entre acteurs et contextes, aux théories de l'individualisme méthodologique, puis à l'approche structurale: place individuelle des acteurs, analyse en termes de réseaux sociaux, structuration de liens individuels entre protagonistes qui relient des actions multiformes, efficacité d'une action collective qui doit beaucoup à l'engagement individuel de certains fonctionnaires.
\end{abstract}

En décembre 2005 fut signé un accord collectif prenant la forme d'un avenant à la convention collective nationale des transports routiers et activités auxiliaires de transport (CCNTR dans la suite du texte). Cet avenant pose un ensemble de règles distinctes pour les activités de la course urbaine, qui ne faisait jusqu'alors l'objet d'aucune disposition spécifique. En droit, le monde de la course ne dépendait donc que du Code du travail. Cette signature, aboutissement d'un long processus de négociation entamé cinq années auparavant, est d'abord la manifestation d'une capacité des représentants des employeurs et des salariés du monde de la course urbaine à élaborer de manière autonome les règles collectives de la profession. D'un point de vue formel, l'avenant coursier représente l'acte fondateur de la constitution d'une branche, affirmant les spécificités de cette activité à l'égard des nombreuses activités de transport routier.

Cet épisode témoigne plus généralement de l'actualité de la régulation de branche, y compris dans des secteurs qui n'y sont pas "prédisposés». En effet, la course urbaine est un secteur symptomatique des dynamiques contemporaines de restructurations productives. Le fort développement de ce secteur au cours des vingt dernières années résulte principalement des politiques d'externalisation de services peu qualifiés. La course est en ce sens le

\footnotetext{
* Clersé, université de Lille 1; marnix.dressen@univ-lille1.fr

** GRIS-IDHE, université de Rouen; mias@univ-rouen.fr
}

produit d'une recherche généralisée de flexibilité externe. Elle témoigne aussi du développement d'une flexibilité interne exacerbée. Longtemps sans cadre régulateur pertinent, l'activité de course s'est développée de façon à maints égards problématique, conduisant à une situation qui articule une faible protection des salariés, une accidentologie jugée par certains élevée et une part non-négligeable d'activité illégale, conduisant à une situation de dumping social et de concurrence par une réduction des coûts du travail (Dressen, Mias, Vacher, 2007).

Au regard de ces divers éléments caractéristiques de la course, la signature d'un avenant coursier à la CCNTR laisse le sociologue des relations professionnelles assez démuni. Ce dernier se trouve en effet mal équipé avec sa boîte à outils conceptuelle traditionnelle. Les données structurelles de contexte, l'état des acteurs collectifs et l'idéologie ne sont effectivement pas favorables à l'élaboration d'une régulation collective. Du moins manque-t-il quelques mécanismes causaux pour expliquer que les acteurs porteurs d'un projet de régulation l'aient in fine emporté à la fois sur les caractéristiques structurelles de ce monde professionnel et sur les acteurs les plus réfractaires à ce type d'initiative.

L'enquête sociologique révèle une pluralité, voire un enchevêtrement de causes, parmi lesquelles l'action publique occupe une place importante. Un ensemble d'institutions et d'acteurs qui n'ont pas les mêmes fonctions, n'interviennent pas au même niveau et dont les initiatives sont parfois indépendantes, voire contradictoires, témoigne d'une présence inter- 
mittente, multiforme, mais néanmoins décisive des autorités publiques dans le processus. C'est à la mise au jour de cette présence de l'État dans les relations professionnelles qu'est consacré cet article, interrogeant la cohérence et l'homogénéité de ces actions.

Il convient de se demander plus précisément par quels mécanismes l'action publique influence l'institution des branches professionnelles en France. Formulée ainsi, la question paraît à la fois classique, ambiguë et inféconde. Classique si l'on considère que l'institution est une notion constitutive et fédératrice des relations professionnelles. Ambiguë car cette notion n'en demeure pas moins polysémique, résultat des raffinements des divers institutionnalismes et néo-institutionnalismes. Inféconde car encombrée d'une série d'oppositions convenues, comme celles du changement et de la continuité ou de l'autonomie et de la structure (Duclos, Mériaux, 2008). Malgré cela, la notion d'institution nous paraît incontournable pour rendre compte du travail de l'État dans les relations professionnelles. En un sens très général, elle peut être appréhendée comme la cristallisation de manières de faire, de penser et de sentir qui se manifeste par la fixation de règles conventionnelles et légales, et par l'établissement concomitant de croyances et de représentations qui donnent sens et effectivité à ces règles (cette dimension symbolique et cognitive est soulignée fermement dans Jobert, SAgLio, 2004).

Dans la compréhension de cette institution, nous souhaitons donner toute sa place à ce qu'A. GidDENS (1987, p. 75) appelle la «dualité du structurel», autrement dit le caractère simultanément contraignant et habilitant des institutions en place: «Selon la dualité du structurel, les propriétés structurelles des systèmes sociaux sont à la fois le médium et le résultat des pratiques qu'elles organisent de façon récursive $»$. De cette vision circulaire des institutions découle le principe selon lequel le structurel ne peut être saisi que dans les actions et interactions. Dans le cas de la course urbaine, les structures politiques interviennent sous la forme d'une action publique qui s'inscrit dans une temporalité singulière, qui fait intervenir une multitude d'acteurs dont les initiatives se composent pour donner un résultat qui ne correspond pas nécessairement aux objectifs poursuivis par chacun de ces acteurs.

Une telle perspective permet de compléter les approches de la régulation sociale qui saisissent l'action de l'État dans les relations professionnelles du point de vue d'une pluralité de modes d'intervention, allant de la facilitation à la substitution (REYNAUD, 1980). Elle pointe une action moins directe mais pourtant décisive sur la structure même $\mathrm{du}$ système d'action des organisations collectives. Notre hypothèse est que les différentes initiatives qui se sont effectivement succédées depuis une dizaine d'années ont construit et stabilisé, progressivement et sous différents aspects (structural et cognitif), le cadre des relations entre employeurs et salariés de la branche, sans que ce résultat réponde à un plan rationnel pré-établi.

L'étude de l'institution de la course urbaine implique alors de décentrer le regard des seuls processus de négociation collective pour envisager l'action décisive et multiforme des autorités publiques dans la dynamique de constitution d'une régulation de branche. Les différentes initiatives que nous allons présenter donnent à voir la pluralité des formes que prend l'action publique dans les régulations du travail. La première partie décrit le système de relations professionnelles de la course urbaine, en analysant la structuration de chacun des trois acteurs: les salariés, les employeurs et l'État. La deuxième partie rend compte des diverses interventions des autorités publiques qui ont favorisé la négociation de l'avenant. La troisième partie pose des éléments d'interprétation de ces développements, en les situant à l'égard des études existantes sur le rôle de l'action publique dans la dynamique des relations professionnelles.

\section{Le système de relations professionnelles de la course urbaine}

D'après les estimations officielles, le secteur de la course concernerait 11000 personnes environ, dont 5000 en région parisienne, et entre 1200 et 1500 entreprises, dont plus de la moitié en région parisienne (RECEVEUR, 2004). Il s'inscrit dans une branche, les transports routiers, où les acteurs collectifs sont faiblement implantés et divisés, et où prédominent au mieux des relations de type paternaliste entre employeurs et salariés; elles sont souvent beaucoup plus dures. Avec la place stratégique qu'occupent les transports dans l'économie nationale, cette présence lacunaire des organisations collectives explique dans une large mesure le rôle traditionnellement important de l'État dans les transports routiers. À cet égard, la course urbaine paraît développer ses relations professionnelles de façon «homothétique», projetant certaines caractéristiques générales du transport routier sur ce monde professionnel en construction. Dans un marché où les résultats des entreprises de course dépendent très largement des relations qu'ils entretiennent avec les donneurs d'ordre, les facteurs salariaux (le jeu avec la masse salariale) l'emportent largement sur les facteurs organisationnels (rationaliser les déplacements des coursiers) dans la détermination de la compétitivité des entreprises. Même si quelques entreprises atteignent une taille importante (plus de 100 salariés), les menaces de concentration du secteur sont faibles, certaines entreprises ayant même tendance à sous-traiter une partie de leur activité (CNR, 2003). 


\section{Salariés et syndicats de salariés}

On dénombre très peu de syndiqués parmi les coursiers, y compris pour la plus importante fédération syndicale, la CGT. Si le tissu des entreprises, largement composé de TPE, explique dans une large mesure les difficultés de syndicalisation, il faut aussi tenir compte des conditions d'emploi et de travail dans l'activité de course. D'abord, les coursiers paraissent peu nombreux à faire carrière dans ce secteur. Au-delà du noyau dur de coursiers qui, bon gré mal gré, restent coursiers de nombreuses années, quitte parfois à papillonner d'une entreprise à l'autre, un volant de coursiers dont la proportion est difficile à évaluer ne conçoit cette activité professionnelle que comme un «job» rémunérateur, sans qu'ils ne se projettent davantage dans l'avenir, l'activité étant au demeurant nerveusement fatigante et exposant les coursiers à nombre de nuisances, à commencer par les intempéries, la pollution et les rythmes de travail. Ce sont de jeunes salariés, souvent issus des banlieues, très faiblement qualifiés (il n'y a pas de formation exigée), qui trouvent dans cette activité une rémunération relativement élevée (au regard de leur niveau scolaire), au prix d'une prise de risque importante dans la circulation urbaine. Toutefois, d'après un représentant patronal, cette situation évoluerait actuellement, le turnover ayant tendance à diminuer de façon marquée.

Les conditions d'une contestation collective de cette situation par les salariés ne paraissent par ailleurs pas réunies. Le collectif de travail est peu présent dans l'activité quotidienne, l'essentiel des interactions passant dans le face-à-face à distance entre le coursier et le dispatcheur (distributeur des courses aux coursiers), aussi appelé régulateur. À cela s'ajoute ce qu'il faut bien envisager comme une culture professionnelle des coursiers, centrée sur une valeur d'indépendance. Indépendance visà-vis des institutions sociales (sentiment de s'être fait soi-même, pour des individus pour la plupart sans diplôme et sans qualification) et vis-à-vis des employeurs: le coursier est fréquemment propriétaire de son véhicule; il a peu de contacts avec son employeur, passant son temps de travail seul et à l'extérieur (avec une certaine autonomie dans son travail, notamment dans le choix des trajets). De nombreux acteurs interviewés nous ont fait part aussi d'une certaine indépendance dans la relation d'emploi et d'un important absentéisme(1). Les conditions de

(1) Les employeurs éprouvent des difficultés pour organiser une régularité du temps de travail: «La course est un pôle d'intégration sociale où le gars peut venir avec son scooter et trouver un métier. Et c'est ce qui justifie son mode de rémunération. C'est-à-dire: le PSG gagne, je me bourre la gueule, je ne viens pas travailler le lendemain; le PSG perd, je me bourre la gueule et je ne viens pas travailler le lendemain. On est face à une population à laquelle, au départ, on ne peut pas appliquer les règles sociales. Tu bosses, t'as des sous; tu bosses pas, t'as pas de sous. La règle est établie comme cela et elle correspond à la psychologie des coursiers» (entretien avec un employeur). travail des coursiers sont ainsi caractérisées par une accumulation d'arrangements individuels destinés à rendre le travail un peu attrayant pour les salariés, mais qui rendent également le contrôle et la prévisibilité difficiles pour les employeurs. L'extension de son espace d'intervention rend par exemple le salarié plus difficile à contrôler et c'est d'ailleurs un des facteurs d'attraction qu'exerce cette activité sur des salariés heureux de «ne pas avoir de patron sur le dos». Le fait d'être propriétaire de son propre véhicule et de ne pas travailler avec un uniforme (à la différence des livreurs dans la restauration) participent également de ces arrangements individuels qui renforcent l'indépendance subjectivement vécue par les coursiers.

Cette valeur d'indépendance est elle-même renforcée par les possibilités de carrière dans la course, secteur fortement marqué par une pratique d'essaimage des entreprises qui conduit les coursiers à créer leur propre société. À cet égard, la course urbaine n'est pas très éloignée, socialement et culturellement, du monde des travailleurs indépendants. Elle s'inscrit bien dans le monde du transport routier, dont l'argumentation professionnelle s'est constituée contre les professions à statut, particulièrement celle des chemins de fer. C. Dubar, P. TRIPIER (1998) ont souligné le fait que l'idéologie du libre marché constitue un ciment idéologique entre les camionneurs et leurs patrons, autour de valeurs individualistes, d'accomplissement de soi et de liberté seulement réglée par le marché, et autour de la figure de l'homme qui s'est fait tout seul. Notons d'ailleurs que la barrière à l'entrée dans le métier de la course urbaine est constituée par le seul permis de conduire des deux-roues.

Dans une telle situation, les syndicalistes conduits à négocier l'avenant coursier entretiennent des relations plus ou moins éloignées avec le monde de la course. Par exemple, si les représentants FO et CGT sont des coursiers, le représentant de la CFDT est un permanent de la fédération, issu lui-même du transport ambulancier, qui est certainement de tous le plus expérimenté, ayant participé à la négociation de différents avenants pour des activités spécifiques de transport. De façon générale, le comportement des salariés paraît exercer une influence sur les stratégies de négociation des organisations syndicales. Sans être à l'initiative de la régulation, la CFDT répond aux initiatives des autres, dans l'objectif d'assurer une couverture conventionnelle des salariés en adoptant une posture pragmatique à l'égard des propositions patronales. La CGT a été plus offensive en demandant, bien avant l'ouverture des négociations, un rendez-vous auprès du cabinet de Jean-Claude Gayssot, ministre communiste des Transports dans le gouvernement Jospin, et accompagnant ensuite des actions devant les prud'hommes (voir infra, p. 12). La dimension collective de l'action syndicale passe ici par une mobilisation du droit visant une accumulation de cas individuels. 


\section{Employeurs et syndicats patronaux}

Les employeurs de la course sont pour la plupart artisans ou à la tête de très petites entreprises. Ils appartiennent à un milieu patronal complexe qui se compose pour partie, et pour partie seulement, de réfractaires à toute régulation collective, qui plus est légale, de leur activité. Mais le monde des travailleurs indépendants est également composé de segments qui visent à réguler les entrées dans la "profession», ainsi que les comportements économiques. Parmi eux, certains soutiennent une intervention de l'État dans le strict domaine économique, d'autres sont partisans d'une régulation plus large, couvrant les conditions de travail.

Fruit de ces divisions, la représentation patronale relève d'une architecture relativement complexe. Trois fédérations d'employeurs se côtoient: l'Union nationale des organisations syndicales des transporteurs routiers automobiles (UNOSTRA), la Fédération des entreprises de transport et logistique de France (TLF), créée en 1998 et la Fédération nationale des transports routiers (FNTR). Les deux derniers coopèrent dans le cadre d'un organisme appelé l'Union des fédérations de transport (UFT), chargé des questions sociales (2). Cet organisme, sans être une confédération, est une fédération de fédérations. Les choses se compliquent encore si l'on tient compte du fait qu'au sein de TLF, par exemple, on trouve des adhérents directs (des entreprises), mais également des syndicats professionnels qui adhèrent à la fédération (en particulier pour pouvoir négocier un accord collectif). Ainsi la négociation collective se fait par le biais de l'UFT, pour le compte de la FNTR et de TLF, d'un côté, et de l'UNOSTRA de l'autre. Une quatrième fédération patronale, l'Organisation des transports routiers européens (OTRE), créée en 2000, n'est pas considérée comme représentative, bien que fédérant un nombre important d'employeurs. Les rivalités sont importantes entre ces différentes fédérations.

Dans le secteur de la course urbaine, le principal syndicat professionnel est le Syndicat national des transports légers (SNTL). En 1997, ce syndicat est moins que vivotant (3). Une équipe formée d'employeurs qui ne sont pas issus du sérail de la course prend alors la direction de ce syndicat, avec d'emblée un projet de régulation et de lutte contre l'illégalité et la concurrence par les coûts du travail(4). Côté

(2) De l'aveu d'un représentant patronal, «ce sont deux sœurs ennemies qui savent que si elles ne se mettent pas d'accord, ce sera difficile $»$.

(3) Le bail du local n'est pas renouvelé. Le délégué général est en arrêt pour longue maladie. On ne sait plus qui adhère.

(4) «Et l'idée de clarifier les règles du jeu était justement de dire: à partir du moment où les règles sont claires, ceux qui ne sont pas dedans, on les fait sortir ou on les fait punir, jusqu'à ce qu'ils se mettent dedans pour éviter la concurrence sur les prix qui est préjudiciable au système» (président du SNTL depuis 1998, interviewé le 16 février 2007). On trouve chez cette personne un attachement manifeste à un assainissement des conditions de la concurrence. patronal, c'est cet acteur collectif qui est le porteur du projet de régulation du monde de la course. Il prend, à bien des égards, la figure d'un «entrepreneur de morale» (BECKER, 1985), catégorisant un ensemble social de déviant (le travail au noir), luttant pour la reconnaissance publique de cette catégorie, cherchant à convaincre de la nécessité morale de nouvelles normes (la lutte contre la concurrence déloyale) et participant enfin à l'élaboration et à l'application des normes auxquelles ces déviants ne se conforment pas.

La représentation des entreprises de la course est très indirecte: lors de la négociation de l'avenant coursier, le président du SNTL a pu siéger à la commission paritaire par le fait que son syndicat adhère à TLF (5), elle-même adhérente à l'UFT. Cela implique une obtention de compromis au sein de la délégation patronale, selon un processus qu'on pourrait qualifier de négociation «à tiroirs》 (négociations au sein de TLF, au sein de l'UFT, puis avec l'UNOSTRA). Nous montrons plus loin que l'État a joué un rôle décisif dans le soutien à ce projet de régulation porté au départ par un acteur patronal agonisant et très isolé. Les raisons pour lesquelles des responsables patronaux acceptent de s'allier à des institutions publiques mêlent tout à la fois des préoccupations morales et des enjeux proprement économiques de discipline du marché (encadrement de la concurrence et lutte contre le travail illégal). Face à ces responsables patronaux, il n'y a pas véritablement d'adversaires à combattre, mais bien plutôt une indifférence, voire un mépris explicite (6), ainsi qu'une crainte d'une remise en cause des équilibres existants : l'intégration des pratiques et des niveaux de rémunération de la course dans l'architecture de la CCNTR induit le risque de déstabilisation des échelles de salaires dans les secteurs proches, notamment dans les grands groupes de la messagerie express. Autrement dit, le projet de régulation a été porté dans le monde patronal des transports routiers par un représentant de petites entreprises, contre les réticences de grandes entreprises, ce qui témoigne de l'attitude singulière des petites entreprises à l'égard de la négociation collective. En sus de la volonté de réguler les relations de concurrence entre TPE, l'objectif poursuivi par les représentants patronaux est d'équiper les relations de travail et d'emploi dans la course: dans ce secteur à l'instar d'autres branches de petites entreprises (SAGLIO, 2006), la négociation collective est une ressource pour les PME car elle leur fournit entre autres un résumé présentable du Code du travail, une norme de justice auxquelles

(5) Lors de la création de TLF en novembre 1998, le SNTL choisit de quitter la FNTR pour adhérer au nouveau syndicat patronal.

(6) Que nous rapporte le président du SNTL: «On me répond que le transport léger est une erreur de l'histoire», «on me demande ce que les mobylettes et les livreurs viennent faire dans la convention collective» (entretien du 16 février 2007). 
employeurs et salariés peuvent se référer dans leur relation d'emploi, ou encore un guide en ce qui concerne l'organisation du travail, via la description des différents postes dans la classification.

\section{État et institutions publiques}

Les relations de l'État avec les organisations syndicales de la branche des transports sont elles aussi très singulières (seule l'agriculture paraît s'approcher de ce modèle de relations). On y observe effectivement une activité soutenue et régulière en matière de réglementation, de contrôle et de soutien à la négociation collective, dont les fondements ont été posés au lendemain de la Seconde Guerre mondiale, lors de la période de reconstruction. Une inspection du travail des transports se spécialise alors dans le contrôle de l'application des normes légales dans ce secteur. Dans le domaine de la négociation collective, la mise en place de commissions paritaires est prévue par la CCNTR de 1950. Les partenaires sociaux expriment dès cette date le souhait qu'un fonctionnaire préside la commission nationale de conciliation et d'interprétation de la convention collective. Cette présidence n'a, depuis lors, jamais été remise en cause. Les réunions entre partenaires sociaux sous l'égide de représentants $\mathrm{du}$ ministère sont régulières et très fréquentes. Le ministère exerce également un rôle important de veille à l'égard des conflits sociaux, pour prévenir le blocage des routes et des voies ferrées. Ainsi, à l'issue des réunions, un compte rendu est systématiquement envoyé au cabinet du ministre et diffusé au sein de l'Inspection du travail des transports.

Les transports pourraient se prêter à une lecture néocorporatiste: la présence traditionnellement forte de l'État dans le soutien aux négociations et la légitimation des organisations collectives répondent à la volonté de prévenir et de limiter les conflits sociaux qui ont un impact global sur l'économie du pays. Cette fonction s'avère décisive dans un secteur (les transports routiers plus spécifiquement) où employeurs comme salariés sont rétifs à toute organisation collective de la profession. Du point de vue du ministère, «les organisations, bien que peu implantées en France, sont des relais des difficultés des salariés ou des entreprises » (directeur du travail, chef du bureau de la négociation collective, des statuts et de la représentation du personnel, entretien du 26 avril 2006).

Au-delà même de ce soutien traditionnel aux organisations représentatives du secteur, la présence de l'État dans cette branche est très spécifique. En effet, rares en France sont les secteurs où le même ministère assure à la fois le contrôle économique, technique et social de la profession, y compris au niveau de l'Inspection du travail des transports qui ne dépend pas de l'autorité du ministère du Travail. Autrement dit, le ministère en charge de la régulation sociale est celui qui concentre l'ensemble de l'action de l'État dans le secteur.

\section{L'action des autorités publiques dans l'institution d'une branche}

Cette place traditionnellement forte de l'État dans la régulation des transports routiers n'implique pas nécessairement une mise en forme légale-rationnelle des régulations dans les branches nouvelles de transport. C'est même plutôt l'inverse que l'on observe dans le cas de la course urbaine.

Et cela se manifeste au niveau élémentaire de la définition de l'identité des sociétés de course, à travers deux initiatives: l'inscription des entreprises de la course urbaine au registre des transports (via l'extension de la loi d'organisation des transports intérieurs (LOTI) à ces entreprises), qui de facto, entre autres conséquences, les fait entrer dans le champ de compétence de l'Inspection du travail des transports (et non plus de l'Inspection du travail générale); le rattachement de la course à la CCNTR. Il s'agit d'éléments fondamentaux car ils déterminent l'existence même de la branche et son périmètre. Dans cette perspective, il faut décentrer le regard à l'égard des seules régulations du travail pour tenir compte de la construction de l'identité économique du secteur et des régulations qui y sont associées. Ici comme là, le rôle de l'État et de sa bureaucratie est décisif, sans toutefois prendre la forme d'une action pleinement rationnelle. Il s'agit en effet de deux actions indépendantes, qui toutes deux relèvent de la compétence étatique. C'est la non-coïncidence de ces deux initiatives qui, dans le cas de la course, nous fait pointer la rationalisation incomplète de l'action publique dans la régulation de branche.

\section{Les incertitudes dans l'identification de la branche}

La manière dont l'État a conçu son rôle dans la réglementation de l'activité de course donne à voir une intervention progressive, croissante et continue depuis environ un quart de siècle. En effet, à partir de 1982, seules les entreprises possédant des véhicules d'au moins deux essieux de plus de 3,5 tonnes de PTAC sont concernées par la toute nouvelle LOTI, qui les oblige à s'inscrire au registre des transporteurs et donc à respecter les conditions d'honorabilité et de capacités professionnelle et financière. En 1998, à la faveur de son actualisation, la loi «Gayssot» (qui actualise la LOTI) est étendue aux entreprises possédant des véhicules à deux essieux de moins de 3,5 tonnes. Du coup, la situation des coursiers en deux-roues dépend de cet unique critère: l'entreprise possède-t-elle des véhicules à quatre roues? Dans l'affirmative, tout son personnel, qu'il travaille en deux-roues ou en quatre roues, dépend du ministère des Transports et de son inspection du travail spécifique. Mais les entreprises qui n'emploient que des deux-roues ne sont pas tenues à l'inscription au registre des transports. Elles relèvent banalement du ministère du Travail et de son inspection ordinaire. 
Ce n'est qu'en décembre 2005, en l'absence d'une initiative de l'État, qu'un amendement parlementaire arraché par l'acteur patronal allié à des hauts fonctionnaires étend la LOTI à toutes les entreprises de courses, qu'elles emploient ou non des quatre roues (7). Ainsi se termine une période d'environ trois décennies au cours de laquelle des entreprises n'employant que des coursiers en deux-roues ont échappé à toute réglementation spécifique.

$\mathrm{Au}$ plan conventionnel maintenant, il est assez surprenant de constater qu'à la veille de l'été 1998 toute la course, quelle que soit sa situation à l'égard de la LOTI, quels que soient ses outils de travail, a été rattachée à la CCNTR datant de 1950. Que peut-on dire de ce processus? Qu'il est constitué de diverses initiatives: d'abord d'une démarche de la CGT des coursiers en direction du ministère des Transports alors confié au ministre J.-C. Gayssot, membre du Parti communiste. La CGT se plaignait de ce que les coursiers ne bénéficient d'aucune couverture conventionnelle et relèvent donc seulement du Code du travail. En réalité, au dire d'un des principaux acteurs patronaux, les plus importantes entreprises de courses se référaient spontanément à la convention collective des transports routiers. Mais elles n'en étaient pas satisfaites car ce contrat collectif était manifestement inadapté au transport léger, ne comptant aucune disposition qui lui soit propre. C'est dans ces conditions que l'équipe dirigeante du SNTL a entrepris une démarche en direction du ministère du Travail pour l'interroger sur le rattachement de la course à une convention collective existante et pour souligner, dans tous les cas, l'inadaptation de la CCNTR au cas de la course urbaine. Mais, s'abstenant de formuler une opinion, le haut fonctionnaire qui les a reçus ne semble pas dans les semaines qui ont suivi avoir arbitré en faveur du SNTL. En tout cas, du fait de son intervention, en juillet 1998, un décret annonce que la course est désormais rattachée à la CCNTR.

Mais cette décision ministérielle aboutit à une situation hybride et à maints égards peu cohérente: les entreprises qui n'emploient que des deux-roues relèvent d'une convention collective dont le ministère des Transports assure le suivi, mais ne dépendent pas du même ministère du point de vue de leur réglementation et du corps d'inspection du travail. Le président du SNTL présente ainsi les incohérences du traitement politique de la course: «On n'est pas du monde du transport, mais par contre on applique la convention collective.» (Président du SNTL, entretien du 16 février 2007).

(7) D'après le président du SNTL, cet amendement était combattu au sein même de l'État par la direction régionale de l'équipement d'Île-de-France avec le soutien du ministre car la DRE n'avait pas le personnel nécessaire pour assurer ce contrôle.
Une telle situation a permis à des employeurs peu scrupuleux de développer une activité en marge de la loi, pratiquant le dumping social(8). Certes, depuis 1998 tous les salariés de la course dépendaient bien de la CCNTR. Mais celle-ci ne contenait aucune disposition spécifique relative aux coursiers, et au vu de la balkanisation de la sous-branche, du manque de moyens de l'inspection du travail ordinaire (9) et de l'extrême faiblesse d'implantation syndicale qu'elle connaît, aucun acteur n'était en mesure de faire respecter la loi, si ce n'est de temps à autre, la justice prud'homale.

Le rattachement à une branche professionnelle a donc constitué une première étape de la régulation conventionnelle. Les employeurs de la course se sont vus imposer par arrêté du ministère du Travail le cadre conventionnel dans lequel ils devraient désormais développer leurs activités économiques et, dans le registre des relations professionnelles, leur activité d'organisation patronale.

Le rattachement doublement problématique à la CCNTR et à la LOTI représente de toute évidence une évolution du contexte qui a poussé l'acteur patronal, alors en pleine mutation, à s'engager dans la voie d'une négociation d'un avenant coursier spécifique à la CCNTR, tout en militant parallèlement pour l'extension de la LOTI à l'ensemble des sociétés de course, y compris celles qui n'emploient que des deux-roues. Cette construction politique du contexte est une première manifestation d'une action publique contingente dans la branche. Cette action dessine un enjeu décisif pour l'engagement patronal dans la négociation collective. Très habituellement en effet, la première question à traiter a consisté à délimiter précisément et pratiquement les contours de la branche en question(10). Cette question est tout à la fois le moteur de l'engagement patronal et la source des blocages au sein de la délégation patronale pendant les premières années de la négociation. L'émergence de cet enjeu paraît le résultat involontaire d'actions ministérielles incohérentes. Cette incohérence manifeste n'est pas ici le fruit de dynamiques administratives non-concer-

(8) Selon un rapport officiel émanant du ministère de l'Équipement et des Transports: "Compte tenu des exigences des donneurs d'ordres, les tarifs sont tirés vers le bas, au prix d'un mépris flagrant des lois sociales, voire de la sécurité et même de la pérennité de ces entreprises » (RECEVEUR, 2004, p. 4).

(9) Dans la mesure où les entreprises de courses étaient et demeurent très nombreuses et n'emploient que peu, voire très peu, de salariés, la probabilité d'un contrôle était extrêmement réduite.

(10) Tel qu'il apparaît au final dans l'avenant à la CCNTR, le champ d'application propose une première définition de l'activité de course: l'acheminement de plis, colis ou objets, sans rupture de charge, la prise en charge et la livraison de chaque marchandise ayant lieu dans une même zone urbaine et/ou périurbaine, au moyen de véhicules deux-roues. L'avenant s'applique également aux entreprises exploitant à titre principal des véhicules deux-roues, lorsque les personnels coursiers sont affectés sur tout véhicule jusqu'à 3,5 tonnes de PTAC. 
tées relevant de ministères différents, comme cela pourrait être le cas dans d'autres branches, mais bien plutôt de la non-congruence de différentes interventions politiques visant à définir les marges du périmètre administratif de deux champs de compétence du ministère des Transports, la LOTI et la CCNTR. Le mécanisme causal à l'œuvre ne relève donc pas d'une mécanique administrative classique, mais bien d'un cas spécifique d'intervention politique dans un contexte particulier, marqué par l'action volontariste du ministre Gayssot(11).

\section{Les interventions multiformes des autorités publiques dans la négociation collective}

Dans le processus qui conduit à l'ouverture d'une négociation collective et jusqu'à la signature de l'avenant (et même au-delà), l'action de l'État se révèle encore très présente et multiforme. À côté des interventions diverses du gouvernement et de son administration, deux autres institutions publiques ont exercé une influence non-négligeable sur le processus en développant notamment l'agenda des négociations, en accélérant par moments le rythme des négociations et en pesant plus généralement sur les motifs d'action des syndicats (d'employeurs et de salariés) et leur volonté d'aboutir dans un délai raisonnable à un texte qui pose un cadre normatif pertinent.

Les collectivités territoriales, ici la mairie de Paris, représentent un acteur peu fréquent dans le domaine des relations professionnelles (même si leur rôle semble se développer dans le cadre du dialogue social territorial (JOBERT et al., 2008)). Si son influence sur les négociations collectives est indirecte, la Mairie de Paris n'en contribue pas moins à en fixer certains enjeux. Son intervention répond avant tout au souci d'assurer une plus grande sécurité sur la voirie municipale, ce qui est de sa responsabilité. Elle conduit à des campagnes de sensibilisation sur ces questions, faisant intervenir des acteurs d'horizons très différents (pompiers, Caisse régionale d'assurance-maladie d'Île-de-France, préfecture de Police, associations de motards, aux côtés des syndicats d'employeurs et de salariés).

Cette initiative municipale a surtout contribué à fixer un enjeu autour de l'accidentologie problématique des deux-roues, qui a conduit le SNTL à demander le développement d'une expertise publique dans ce domaine, pour mettre en doute non seulement la validité de ce constat, mais surtout le lien de causalité (selon lui trop vite) fait avec le mode de rémunération. On observe donc ici une problématique d'ordre public qui s'arti-

(11) Plus généralement, la part d'initiative voire de fantaisie des acteurs est parfois importante. Les hauts fonctionnaires n'appliquent pas des règles, ils les inventent pour une part lorsqu'une décision à prendre se présente. cule avec d'autres, notamment le droit du travail. Cette initiative a eu pour effet de renforcer les liens entre l'acteur patronal, la CNAM, la CRAMIF et l'administration centrale, avec le développement d'une expertise spécifique qui va avoir pour conséquence inattendue, si ce n'est l'élaboration, du moins la consolidation de l'agenda des négociations collectives. Deux rapports élaborés en 2004 (VAN Belleghem, Bourgeols, 2004; Receveur, 2004) clarifient les enjeux de la négociation collective.

Ces deux études, soutenues sur le terrain par le SNTL, permettent aux acteurs, publics et sociaux, de développer un constat commun sur les réalités du secteur, facilitant grandement la conduite et l'aboutissement des négociations. L'initiative de la mairie de Paris intervient ainsi dans la phase cruciale où se fixent les enjeux de la négociation, où se formulent les problèmes à régler. J.-D. REYNAUD (2001) insiste sur le caractère décisif de cette phase de toute négociation. Lui accordant un primat méthodologique, il incite à porter un regard attentif aux dimensions cognitives du processus. C'est sous cet angle effectivement que l'initiative municipale nous paraît décisive (12).

Une autre institution publique a contribué à fixer l'agenda des négociations collectives en cours: la justice prud'homale. On peut même y voir un accélérateur du processus de négociation avec la condamnation de pratiques de rémunération jugées abusives. Cette jurisprudence est le résultat d'une mobilisation systématique de l'instance prud'homale par des salariés soutenus par le syndicat CGT des coursiers, qui aide à la constitution des dossiers et de la plaidoirie. À plusieurs reprises, les plaignants ont pu montrer que la rémunération des heures supplémentaires était fautive. Faute de rédiger correctement les feuilles de paie, certaines entreprises ont été lourdement condamnées. Cette bataille juridique accompagne une stratégie d'implantation du syndicat dans les entreprises de la course. Elle conduit à des condamnations symboliquement fortes et financièrement élevées (des entreprises ont dû déposer leur bilan du fait de condamnations pour rappel d'heures supplémentaires), qui marquent les esprits patronaux et posent l'urgence d'une clarification concernant le mode de calcul du temps de travail et des rémunérations.

Le ministère des Transports et de l'Équipement est lui aussi présent dans la négociation collective à

(12) Notons au passage que ces rencontres ont débouché sur une charte de bonnes pratiques des deux-roues motorisés, signée le 19 mars 2007 entre le maire de Paris et les associations concernées, notamment la Fédération française des motards en colère, la Fédération française de motocyclisme... mais également le syndicat CGT des coursiers. Cette charte entend apaiser les relations entre deux-roues motorisés, automobilistes et piétons, et aborde les thèmes de la circulation, du stationnement, des équipements de sécurité et de l'adaptation à l'environnement urbain. 
travers l'action de son administration. Cette action se manifeste par l'encadrement direct des négociations collectives, tel qu'il est traditionnellement pratiqué dans les transports. Mais elle ne se réduit pas à une action bienveillante pour faciliter les débats.

L'influence du ministère passe aussi par la sollicitation discrète de l'acteur patronal au moment même où une nouvelle équipe prend la direction du SNTL, comme le relate le principal protagoniste: «En mars 1998, X [directeur du travail, chef du bureau de la négociation collective, des statuts et de la représentation du personnel] m'a appelé en me disant: "ce serait bien que les coursiers aient une convention collective, parce qu'aujourd'hui le monde est flou". L'idée n'était pas mauvaise» (président du SNTL, entretien du 16 février 2007). L'action du ministère se manifeste encore pour lever les blocages patronaux lors de l'ouverture des négociations: «Donc $\mathrm{M}^{\text {me }} \mathrm{Y}$ [présidente d'une commission ad hoc au sein de TLF] valide la possibilité de négocier. TLF demande à l'UFT de bien vouloir mettre à l'ordre $\mathrm{du}$ jour la question d'un avenant à la convention collective. La FNTR essaie de bloquer. J'accède très haut au ministère, à un pouvoir qui dit à la FNTR:
"Laissez-les discuter". Et on s'assoit autour de la table et commence une autre aventure, qui est celle de la discussion d'un avenant.» (Ibidem).

Le soutien de l'État apparaît également décisif dans le cours même des négociations. Le fonctionnaire du ministère de l'Équipement et des Transports, chef du bureau de la négociation collective, qui a présidé ces négociations évoque sa fonction comme celle d'un «facilitateur». À cet égard, la tâche n'est pas aisée, tant les points d'achoppement, bien que classiques, ont été nombreux: l'enjeu de la définition du champ d'application (source classique de dissensions au sein de la délégation patronale), le temps de travail et son calcul, les modes de rémunération... Dans l'entretien qu'il nous a accordé, ce fonctionnaire décrit la rédaction de l'avenant comme un «mille-feuilles», procédant d'étape en étape, nécessitant plusieurs fois la redéfinition des mandats de négociation initiaux. D'où le rôle décisif de l'État pour maintenir le cap des négociations, l'objectif commun d'adoption d'un avenant au contenu approprié (voir encadré). C'est à ce niveau aussi que l'expertise publique développée parallèlement à la négociation s'avère cruciale.

\section{Encadré 1}

\section{Les principales dispositions de l'avenant “coursiers" à la CCN des transports routiers et activités auxiliaires de transport}

L'avenant n० 94 du 13 décembre 2005 «relatif aux dispositions particulières aux ouvriers » comprend six articles.

L'article $1^{\mathrm{er}}$ fixe un certain nombre de dispositions concernant les personnels coursiers. Outre la définition du champ d'application, l'accord met en place un système de calcul du temps de travail, avec un carnet de route qui comporte le début et la fin de la journée de travail. L'amplitude journalière est diminuée d'une durée forfaitaire d'une heure pour tenir compte des «périodes d'inaction, de repos, repas, pauses, coupures et de la variation de l'intensité de leur activité ". Ce point est particulièrement litigieux et a fait l'objet d'une contestation forte de la part des organisations non-signataires (CGT et FO).

La question du mode de rémunération est absolument décisive dans une situation où la rémunération au bon (autrement dit, à la course) est très fréquente. Selon l'avenant, la rémunération du coursier se compose de deux parts: une part fixe (taux horaire conventionnel garanti, selon l'ancienneté dans l'entreprise, multiplié par la durée effective de travail) et une part variable définie au sein de l'entreprise. Des minima sont également fixés pour cette part variable: $6 \%$ du taux horaire conventionnel garanti pour un emploi de coursier $1^{\mathrm{er}}$ degré, $15 \%$ pour un emploi de coursier $2^{\mathrm{e}}$ degré. Deux remarques s'imposent. La première est celle de l'introduction d'un taux horaire de base pour l'activité de coursier, qui n'existait pas auparavant, et l'abandon du paiement au bon. La seconde est le constat d'une esquisse de définition d'une carrière de coursier avec une augmentation du taux horaire lorsque le coursier progresse dans la classification à trois niveaux. On tend ainsi à reconnaître une qualification singulière du coursier liée à un apprentissage du métier.

La formation est une question décisive dans la reconnaissance sociale du métier de coursier, en le distinguant bien d'un simple «job» ne nécessitant pas de formation. L'avenant définit une formation initiale obligatoire de trois jours avant d'entrer dans le métier de la course, à laquelle s'ajoute, dans les six premiers mois suivant l'embauche, deux jours de formation complémentaire. Ces formations sont prises en charge par l'OPCA transport.

L'article 2 propose d'introduire une nouvelle classification (très simple) dans les nomenclatures et définitions des emplois des ouvriers des transports routiers de marchandises: coursier, coursier confirmé $1^{\text {er }}$ degré et coursier confirmé $2^{\mathrm{e}}$ degré. Les critères fondant cette classification sont l'ancienneté et l'autonomie dans la réalisation des courses.

L'article 4 fixe un programme de poursuite des négociations, témoignant d'une volonté commune d'approfondir la régulation de l'activité de course. L'avenant apparaît ainsi comme un premier jalon, avant l'ouverture de négociations sur cinq nouveaux thèmes, qui s'inscrivent dans la continuité des préoccupations manifestées lors de la négociation de l'avenant: le carnet de route autocopiant; les frais de déplacement; le contenu et la durée des modules de formation; l'emploi de «dispatcheur " (contenu et positionnement hiérarchique); les moyens d'identification du coursier (uniforme...). 


\section{L'État des relations professionnelles}

Tout en reconnaissant les spécificités de l'action publique dans la course(13), cette étude de cas soulève des questions qui interrogent plus largement la place de l'État dans les relations professionnelles. Au cœur de ce qui manifeste l'autonomie contractuelle des syndicats, la négociation d'une convention collective, l'analyse de processus donne à voir la pluralité des actions publiques qui structurent ces dynamiques de régulation. Comment alors rendre compte de cette influence politique? Chaque action que nous avons décrite, prise isolément, peut être rapportée à la typologie des formes d'intervention de l'État esquissée par J.-D. Reynaud (1980): de la stimulation à la substitution pure et simple, en passant souvent par l'encadrement. Ces catégories d'analyse font voir qu'on n'a pas affaire à deux parties en face à face «surveillé» par l'État, mais plutôt à deux acteurs qui entretiennent des relations bilatérales avec l'État. Et souvent l'État joue un jeu autonome qui excède ce qu'attendent les organisations syndicales: son intervention ne consiste pas seulement à arbitrer, mais il n'est pas rare qu'il tienne la barre ou qu'il menace de légiférer. Face aux dissensions au sein de la délégation patronale, c'est la menace d'une initiative législative qui a plusieurs fois ramené les protagonistes autour de la table.

Toutefois, si elle joue un rôle décisif dans l'institution de la branche, l'action publique ne se réduit pas à une série d'interventions ponctuelles. Elle ne prend sens que rapportée à une dynamique d'ensemble qui témoigne selon nous du rapport entretenu par le politique aux relations professionnelles. La problématique du «tiers acteur» montre qu'il n'y a pas de symétrie pure entre les trois «parties». Elle manque néanmoins la dimension temporelle qui fonde la cohérence a posteriori de ces actions publiques. Cette temporalité singulière et contingente est ce qui leur confère dans le même temps leur caractère constituant pour les relations professionnelles de la branche, au sens où leur succession structure le contexte de négociation des acteurs sociaux. Comme le suggère M. TAllard (2004, p. 29), cette dimension constituante de l'action publique n'est pas évidente à saisir: les analyses de J.-D. Reynaud «permettent de classer les différentes actions de l'Etat, d'une part, et de cerner les dynamiques propres aux acteurs et aux règles qu'ils créent d'autre part [...] mais elles appréhendent plus difficilement les mécanismes par lesquels s'élaborent les relations entre l'État et les acteurs sociaux. Elles ne posent pas la question de savoir comment

(13) Et il faut reconnaître que le rôle de l'État est variable selon les branches et qu'il est souvent d'autant plus interventionniste que les organisations collectives sont faibles ou paralysées. l'action publique "produit" les acteurs et, réciproquement, quelle part prennent ces derniers à l'élaboration de cette action».

Abandonner le prisme décisionniste permet de développer une perspective en termes de travail de l'État (14), qui part du caractère ouvert de ce travail et de la pluralité des institutions qui y participent pour en proposer une compréhension qui donne sa place à la «dualité du structurel». Nous entendons par là que, bien que les actions étudiées puissent relever, à certains égards et ponctuellement, d'une nécessité administrative ou d'une mise en cohérence du droit dans la perspective d'une rationalisation formelle (au sens de M. Weber), leurs conséquences débordent largement ces logiques et leur cohérence d'ensemble n'apparaît que rapportée à une dynamique circulaire non close: «Du cours de l'action surgissent sans cesse des conséquences non voulues par les acteurs et, de façon rétroactive, ces conséquences non intentionnelles peuvent devenir des conditions non reconnues d'actions ultérieures. L'histoire humaine résulte d'activités intentionnelles mais elle n'est pas un projet voulu, elle se soustrait constamment à tous les efforts qui sont faits pour la subordonner à une direction consciente» (GIDDENS, 1987 , p. 76). Pour rendre compte de l'action publique dans les relations professionnelles, nous défendons ainsi un individualisme méthodologique qui ne réduit pas le social à des interactions ou à des jeux stratégiques, mais qui fait de la non-intentionnalité des conséquences et de la non-reconnaissance des conditions de l'action des éléments indispensables à la compréhension du caractère instituant de cette action publique.

\section{Lever l'ambivalence de l'acteur et du contexte politiques}

Cette perspective reformule la problématique traditionnelle de l'acteur et du contexte, et notamment de l'acteur public et du contexte politique. C'est une manière de lever l'ambiguïté fondamentale dans la façon de saisir le politique dans les relations professionnelles. Chez J.T. Dunlop (1958) par exemple, les «systèmes de relations professionnelles» font intervenir l'État, en tant qu'acteur constitutif, au même titre que les organisations syndicales et patronales, mais sous la forme stricte des institutions publiques spécialisées dont la fonction est d'assister les deux acteurs dans leurs relations. Le politique apparaît également dans cette œuvre fondatrice sous la forme d'un contexte qui, à l'instar des contextes technologiques et économiques, structure les relations entre acteurs et les «produits» du système, les

(14) Dans un sens peu éloigné de la notion de «travail juridique» entendu «dans les différents lieux de débats, comme la recherche, par aménagements successifs, d'un texte qui soit acceptable pour la plus grande partie des membres d'une communauté juridique $[. .$.$] , c'est-à-dire qui ne se heurte à$ aucune opposition absolue» (DIDRY, 2002, p. 15). 
règles qui encadrent l'activité de travail. Selon les passages, le «politique» glisse de façon imprécise du statut d'acteur caractéristique du système à celui de contexte, voire d'idéologie globale du système. Une ambiguïté fondamentale tient à la difficulté à distinguer l'acteur politique du contexte politique, à «externaliser la variable politique de contexte»: «Les systèmes de relations industrielles s'avèrent moins "qualifiés" par le politique que "spécifiés" précisément par le type de rapport au politique qu'ils intègrent et développent, créant et construisant leur contexte» (GANNE, 1990, p. 223). Sans aller jusqu'à dire qu'il n'existe pas d'autre «contexte politique» que celui qui est construit dans la dynamique même du système de relations professionnelles, il faut reconnaître la difficulté à réduire le politique à un facteur causal extérieur au système.

Difficile à lever, cette confusion entre contexte et acteur politiques est de notre point de vue plus qu'une ambiguité conceptuelle: un élément inhérent à la vie des relations professionnelles, qui participe de leur dynamique. L'État ne peut être assimilé ni à un environnement légal ou idéologique, ni à un acteur au même titre que les organisations syndicales. Il convient plutôt de reconnaître que les structures politiques n'agissent que par le biais de pratiques empiriquement observables. Ces pratiques, tantôt de simples routines qui reproduisent les structures, tantôt de véritables actions orientées vers une fin significative, exercent des effets sur la vie des relations professionnelles au-delà des intentions des agents.

Le cas de la course urbaine n'est qu'une illustration particulièrement prononcée (15) de cette dialectique générale : l'institution de la branche apparaît ici comme une conséquence non voulue de la convergence contingente d'actions publiques engagées à différents niveaux en réponse à des préoccupations d'ordre divers: ordre public; discipline de la concurrence; politique des transports; périmètre des responsabilités administratives; respect du Code du travail, etc. C'est cette convergence qui structure le contexte d'action immédiat à partir duquel les stratégies des responsables patronaux et syndicaux prennent sens (16).

Par exemple, l'initiative de la mairie de Paris et les actions en justice construisent le contexte de la négociation collective selon des modalités très particulières :

- elles ne relèvent pas d'interventions strictement unilatérales des institutions publiques, soit qu'elles

(15) Ce caractère plus prononcé doit beaucoup aux spécificités de l'action publique dans la branche des transports: les actions de l'État peuvent trouver ici plus facilement qu'ailleurs une cohérence et une homogénéité qui leur confèrent une portée causale particulièrement importante.

(16) On peut noter au passage la difficulté à mobiliser le cadre d'analyse proposé par A. Strauss (1978), fondé sur la distinction entre «contexte de négociation» et "contexte structurel». Ici, le second ne cesse de reconfigurer le premier. enclenchent un processus faisant intervenir les acteurs sociaux eux-mêmes (cas du travail d'expertise engagé à la suite de l'initiative de la mairie de Paris), soit qu'elles soient plus ou moins le résultat de l'action délibérée de l'une des parties à la négociation (cas du travail de mobilisation du droit par le syndicat);

- elles ne répondent pas à la volonté de peser sur les négociations collectives mais conduisent à stabiliser leur agenda;

- intervenant parallèlement aux négociations, elles exercent une influence importante sur leur rythme et favorisent in fine l'aboutissement à un accord collectif.

Si le rattachement doublement problématique à la CCNTR et à la LOTI constitue un contexte déterminant pour l'ouverture des négociations, il paraît évident qu'un des motifs patronaux a été également d'adapter la législation relative au mode de rémunération des coursiers, dans une situation d'urgence construite par une jurisprudence importante et par la mise sur agenda de l'accidentologie élevée des deux-roues parisiens.

\section{Le rôle des individualités: pour une approche structurale des relations professionnelles}

$\mathrm{Si}$, à un niveau global, cette structuration du contexte de négociation paraît relever en grande partie du hasard, la convergence des actions publiques est loin d'être spontanée et s'explique par l'action continue d'individus particuliers. Pour donner sens à la dynamique d'ensemble, il faut envisager derrière les relations entre organisations collectives l'importance de certaines relations interindividuelles. Encore faut-il, là aussi, ne pas sombrer dans un individualisme atomiste qui négligerait l'influence des structures de relations sur l'action (suivant en cela une suggestion de F. Chazel, 1986) et saisir l'action publique dans les relations professionnelles comme encastrée dans une structure de relations sociales suivies et significatives (17).

Dans le cas de la course, ces relations ne doivent pas être vues comme des liens affectifs ou de complicité, mais bien plutôt comme le résultat contingent de positions durables d'acteurs qui facilitent la conduite du «projet» à son terme. C'est en tout cas ce que l'on observe du côté patronal, avec la constitution d'une équipe dirigeante du SNTL, porteuse $\mathrm{du}$ projet de régulation du monde de la course urbaine, qui s'engage dans un long travail d'argumentation au sein de TLF pour acquérir l'autonomie nécessaire à la conduite de la négociation collective. Si l'action publique exerce une influence décisive

(17) L'analyse des réseaux a déjà été mobilisée par la sociologie des relations professionnelles: AbaLLEA et al., 2003; MIAS, 2007. 
sur l'institution de la course, celle-ci doit également beaucoup à l'acteur patronal, plus précisément à un petit nombre de personnes issues du SNTL et de TLF. Outre un important travail de négociation interne au monde patronal pour «apprivoiser les grands patrons », selon l'expression du président du SNTL, le rôle de cet acteur patronal tient à la dynamique des relations qu'il a nouées avec différents segments de l'État (Dressen, Mias, 2008).

Ces liens patronaux avec l'État passent par des relations privilégiées (parce que répétées et inscrites dans la durée) avec quelques fonctionnaires. Parmi ceux-ci, deux individus paraissent occuper une position décisive dans la dynamique relationnelle: un directeur du travail, chef du bureau de la négociation collective au sein du ministère, qui sollicite discrètement la nouvelle équipe dirigeante du SNTL en 1998, facilite les négociations avec les organisations syndicales, participe au déblocage des tensions patronales et alimente les négociations avec des expertises (18); une ancienne inspectrice du travail, devenue conseillère sociale de J.C. Gayssot au ministère, qui, à cette position, reçoit les délégations CGT et SNTL au moment de la LOTI et du rattachement à la CCNTR, puis en 2002, ayant quitté le cabinet ministériel, reçoit mission d'engager une étude sur les coursiers, étude qui va largement contribuer à fixer l'agenda des négociations. Les échanges avec le SNTL au sein du cabinet ministériel à la fin des années 1990 favorisent un climat de confiance pour la conduite de cette expertise, comme le relate le président du SNTL: «Et Z, à peine disparue du ministère, reçoit une lettre de mission du ministre. Et sa lettre de mission était de faire une étude sur les coursiers. [...] Je l'ai fait venir et j'ai appelé les adhérents pour qu'ils lui ouvrent leur entreprise car il faut du courage pour ouvrir à un inspecteur du travail, même si elle dit qu'il n'y aura pas de sanction, pour lui ouvrir tout, y compris les petites choses, les arrangements qu'il peut y avoir dans telle ou telle société.» (Entretien du 16 février 2007).

Du point de vue de l'individualisme méthodologique, au sens premier du terme, ce poids des individualités dans l'action n'étonne guère: pour mener des actions collectives, il faut des décisions individuelles d'acteurs qui sont à l'initiative. Il surprend davantage si on le confronte au discours des institutions, et notamment des administrations publiques françaises, qui éprouvent souvent quelques difficultés à reconnaître ce fait. Les historiens ne seront pas surpris, eux qui soulignent depuis quelque temps l'influence décisive de certaines personnalités dans les politiques du travail, en particulier parmi les

(18) Un tel individu occupe une position d'intermédiarité très élevée puisqu'il préside la plupart des commissions paritaires dans l'ensemble des branches du transport. Il peut à ce titre jouer sur différentes scènes pour obtenir des compromis locaux. réformateurs sociaux, comme l'ont rappelé récemment Chatriot, Join-LAmbert et Viet (2006).

À ce niveau des actions individuelles, les analyses en termes de réseaux sociaux paraissent les plus pertinentes. Au fondement de la régulation de la course urbaine, on ne trouve pas un acteur collectif au sens de J.-D. Reynaud, mais un réseau d'acteurs. Certes, il existe un projet de régulation qui fonde une action collective. Mais cette action est multiforme, fait intervenir un grand nombre d'acteurs et ne répond pas à un plan délibérément tracé. Ce qui fait la cohérence de l'ensemble, et permet à l'action d'atteindre son objectif, c'est l'existence et le maintien d'une structure de liens individuels entre les protagonistes. Toutefois, le schéma d'interprétation des relations État-société que suggère la notion de «réseau de politique publique» (LE GALÈs, THATCHER, 1995), marqué par des relations horizontales (plus que hiérarchiques), des échanges informels et une absence de fermeture, ne permet pas de saisir la centralité des acteurs étatiques qui, dans notre cas au moins, restent décisifs. Contre l'idée que l'État perdrait de sa prépondérance dans les relations professionnelles, le cas de la course urbaine montre que l'efficacité de l'action collective tient à l'engagement singulier de fonctionnaires, qui justement participent au maintien du réseau, quitte parfois à glisser d'une position structurale à l'autre (à l'image de la directrice du travail, membre du cabinet de J.-C. Gayssot qui devient chargée par Gilles de Robien d'une mission d'étude sur la course).

Le structuralisme méthodologique met au jour des positions durables dans un écheveau de liens sociaux. Cet écheveau, résultat de positionnements individuels et d'interactions répétées entre acteurs, est en même temps le produit des structures politiques qui cadrent ces pratiques individuelles. Ainsi, le maintien dans la durée d'un même chef du bureau de la négociation pour l'ensemble des branches du transport terrestre peut être vu comme une conséquence d'un souci de stabilité dans l'encadrement des relations professionnelles, souci découlant lui-même d'un travail de construction d'un enjeu politique central autour de la politique des transports. Cette position durable, produit de manières de faire administratives routinisées, joue une fonction extrêmement importante dans la dynamique d'ensemble en assurant une continuité sans laquelle les négociations ouvertes en 2000 auraient pu rapidement tourner court. Cette fonction est une forme singulière de facilitation politique, que nous pourrions appeler conductivité structurale, en adaptant la notion de «conductivité structurelle» (SMELSER, 1963) à une approche réticulaire et non fonctionnaliste. Elle relève de ces «conditions non reconnues» de l'action, au sens où les structures de politique publique façonnent ainsi les bases relationnelles susceptibles de faciliter ou de freiner la négociation collective. 
Cette étude de cas conduit à souligner l'influence décisive de l'action publique dans le processus d'institution d'une branche professionnelle. Cette action publique est à la fois une activité régulière dont les conséquences ne se laissent pas réduire à telle ou telle décision, un travail continu qui ne s'assimile pas à une addition d'interventions interprétées comme des coups stratégiques se rapportant à un jeu d'acteurs, et une action structurante tout à la fois sur le plan structural, en cadrant les moyens «relationnels» d'accès au politique pour les représentants syndicaux, et sur le plan cognitif, pour alimenter l'expertise des négociations et plus généralement pour poser les bases d'une définition communément admise du contexte qui fonde l'orientation vers la production de règles. Cette étude invite ainsi à dépasser une lecture décisionniste des interventions de l'État dans les relations professionnelles en général, et dans l'institution des branches professionnelles en particulier, et à interroger plus avant et d'une manière dialectique les notions usitées d'acteur public et de contexte politique. 


\section{Bibliographie}

Aballéa F., Bévort A., Gadea C., Lallement M., Trancart D. (2003), «Réseaux et innovations organisationnelles. Une approche par les relations professionnelles », Travail et Emploi, $\mathrm{n}^{\circ}$ 95, pp. 87-99.

Becker H. (1985), Outsiders. Étude de sociologie de la déviance, Paris, Métailié, (première édition 1963).

Chatriot A., Join-Lambert O., Viet V. (2006), Les politiques du travail (1906-2006). Acteurs, institutions, réseaux, Rennes, Presses universitaires de Rennes.

Chazel F. (1986), «Individualisme, mobilisation et action collective», in P. Birnbaum et J. Leca (sous la direction de), Sur l'individualisme, Paris, Presses de la FNSP, pp. 244-268.

Comité National Routier (2003), «Le transport léger: complément d'enquête», Les cahiers de l'Observatoire, $\mathrm{n}^{\mathrm{o}} 197$, septembre.

DIDRY C. (2002), Naissance de la convention collective, Paris, Édition de l'EHESS.

Dressen M., Mias A., Vacher K. (2007), «Flexibilité du travail, segmentation des collectifs et processus de régulation dans les activités de service. Le cas des coursiers », in F. Aballéa et M. Lallement (sous la direction de), Relations au travail, relations de travail, Toulouse, Octarès, pp. 211-218.

Dressen M., Mias A. (2008), «Représenter les employeurs. Itinéraire et actions d'un président de syndicat professionnel», Terrains \& Travaux, $n^{\circ} 14$, à paraître.

Dubar C., Tripier P. (1998), Sociologie des professions, Paris, A. Colin.

Duclos L., Mériaux O. (2008), «Métamorphose de l'institution corporative et crise des politiques de l'intérêt», in L. Duclos, G. Groux et O. Mériaux (sous la direction $\mathrm{de})$, Le politique et la dynamique des relations professionnelles, Paris, LGDJ, à paraître.

Dunlop J.T. (1993), Industrial Relations Systems, Boston, Harvard Business School Press, 1 ${ }^{\text {re }}$ éd. 1958.

Ganne B. (1990), «Les relations industrielles et leur contexte. À propos du politique comme contexte», in J.D. Reynaud, F. Eyraud, C. Paradeise et J. Saglio (sous la direction de), Les systèmes de relations professionnelles, Paris, éditions du CNRS, pp. 219-225.

Giddens A. (1987), La constitution de la société, Paris, PUF (première édition 1984).
Jobert A., SAglio J. (2004), «Ré-institutionnaliser la négociation collective en France», Travail et Emploi, $\mathrm{n}^{\mathrm{o}} 100$, pp. 113-127.

Jobert A., Béthoux É., Guarriello F., Heidling, E., Machu L., Mias A. (2008), Les nouveaux cadres $d u$ dialogue social: l'espace européen et les territoires, PIE-Peter Lang, à paraître.

Le Galès P., Thatcher M. (sous la direction de) (1995), Les réseaux de politique publique, Paris, L'Harmattan.

Mias A. (2007), «Sociabilité bruxelloise et européanisation des élites syndicales. Apports de l'analyse de réseaux à l'étude du dialogue social européen », in $\mathrm{O}$. Costa et P. Magnette (sous la direction de), Une Europe des élites? Réflexions sur la fracture démocratique de l'Union européenne, Bruxelles, Presses de l'université de Bruxelles, pp. 167-184.

Receveur C. (2004), Propositions pour un contrat de progrès dans l'activité de la course, rapport de l'Inspection générale du travail des transports, novembre, 42 pages et annexes.

REynaud J.-D. (1997), «Les sociétés néolibérales et la transformation du rôle de l'État dans la détermination des conditions de travail», in J.-D. Reynaud, Le conflit, la négociation et la règle, Toulouse, Octarès, pp. 147156 (première édition 1980).

Reynaud J.-D. (2001), «Création des règles et régulation sociale», in G. Groux (sous la direction de), L'action publique négociée, Paris, L'Harmattan, pp. 263-281.

SAgLio J. (2006), «La négociation de branche et les petites entreprises $»$, in Le dialogue social dans les petites entreprises. Colloque du 30 juin 2006 - Paris, troisième rendez-vous annuel DARES-DRT sur les relations professionnelles, Dossier du participant, pp. 29-34.

SMelser N. (1963), Theory of Collective Behavior, New York, Free Press.

Strauss A. (1978), Negotiations. Varieties, Contexts, Processes, and Social Order, San Francisco, JosseyBass.

TAllard M. (2004), Action publique et régulation de branche de la relation salariale, Paris, L'Harmattan.

Van Belleghem L., Bourgeois F. (2004), Le métier de coursier et ses pratiques de prévention. Étude ergonomique pour la prévention des risques professionnels, rapport pour le SNTL et la CRAMIF, 65 p. 